\title{
Regulation of an intergenic transcript controls adjacent gene transcription in Saccharomyces cerevisiae
}

\author{
Joseph A. Martens, ${ }^{2}$ Pei-Yun Jenny $\mathrm{Wu}^{3}{ }^{3}$ and Fred Winston ${ }^{1,4}$ \\ ${ }^{1}$ Department of Genetics, Harvard Medical School, Boston, Massachusetts 02115, USA
}

Recent studies have revealed that transcription of noncoding, intergenic DNA is abundant among eukaryotes. However, the functions of this transcription are poorly understood. We have previously shown that in Saccharomyces cerevisiae, expression of an intergenic transcript, SRG1, represses the transcription of the adjacent gene, $S E R 3$, by transcription interference. We now show that $S R G 1$ transcription is regulated by serine, thereby conferring regulation of $S E R 3$, a serine biosynthetic gene. This regulation requires Cha4, a serine-dependent activator that binds to the SRG1 promoter and is required for SRG1 induction in the presence of serine. Furthermore, two coactivator complexes, SAGA and Swi/Snf, are also directly required for activation of SRG1 and transcription interference of SER3. Taken together, our results elucidate a physiological role for intergenic transcription in the regulation of SER3. Moreover, our results demonstrate a mechanism by which intergenic transcription allows activators to act indirectly as repressors.

[Keywords: Intergenic transcription; noncoding RNA; transcription interference; transcription]

Supplemental material is available at http://www.genesdev.org.

Received August 23, 2005; revised version accepted September 19, 2005.

The analysis of genome-wide transcription in many organisms, including bacteria, yeast, Drosophila, Arabidopsis, mouse, and human, has yielded a common, yet unexpected feature. In addition to the transcription of protein-coding sequences, there is also widespread transcription across non-protein-coding regions (for reviews, see Mattick 2003; Morey and Avner 2004; Huttenhofer et al. 2005; Johnson et al. 2005). In humans, such noncoding transcripts have been detected from intergenic DNA, from introns, and from antisense transcription of exons (Chen et al. 2002; Kapranov et al. 2002; Saha et al. 2002; Bertone et al. 2004; Kampa et al. 2004; Cheng et al. 2005). Additional analysis suggests that much of this transcription is regulated and, thus, may itself play regulatory roles (Cawley et al. 2004; Kim et al. 2005). Recent experiments in the yeast Saccharomyces cerevisiae have also provided evidence for extensive transcription of noncoding regions (Hurowitz and Brown 2003; Havilio et al. 2005; Wyers et al. 2005).

One large and broad class of noncoding RNAs that has been studied in prokaryotes and eukaryotes plays direct

Present addresses: ${ }^{2}$ Department of Biological Sciences, University of Pittsburgh, Pittsburgh, PA 15260, USA; ${ }^{3}$ Laboratory of Yeast Genetics and Cell Biology, The Rockefeller University, 1230 York Avenue, New York, NY 10021, USA.

${ }^{4}$ Correpsonding author.

E-MAIL winston@genetics.med.harvard.edu; FAX (617) 432-6506.

Article and publication are at http://www.genesdev.org/cgi/doi/10.1101/ gad. 1367605 roles in the regulation of gene expression (for reviews, see Bernstein and Allis 2005; Storz et al. 2005). These regulatory noncoding RNAs have been shown to function at many different levels of gene expression. In Escherichia coli, >50 small noncoding RNA regulators have been identified (for reviews, see Gottesman 2004; Storz et al. 2005). In eukaryotes, one intensively studied group, short interfering RNAs (siRNAs), has been shown to control both chromatin structure and mRNA stability (for reviews, see Meister and Tuschl 2004; Bernstein and Allis 2005). Other well studied eukaryotic categories include the Xist and Tsix RNAs that are involved in Xinactivation in mammals, and the roX1 and roX2 RNAs that are required for dosage compensation in Drosophila (for reviews, see Andersen and Panning 2003; Bernstein and Allis 2005). In addition to these examples, noncoding RNAs have been shown to regulate virtually all known steps of gene expression from transcription initiation to mRNA translation.

Several studies have suggested a second class of noncoding transcription that plays an important role in transcription regulation. In this second class, it is the act of transcription that confers activation, rather than the RNA product itself (for review, see Morey and Avner 2004). One example of this type of regulation occurs in the Drosophila bithorax complex (BX-C). Several early studies demonstrated that transcription occurs across noncoding regions at $\mathrm{BX}-\mathrm{C}$, suggesting a possible role in 
mediating repression by the Polycomb group (PcG) complexes (Lipshitz et al. 1987; Sanchez-Herrero and Akam 1989; Cumberledge et al. 1990). More recent studies have shown a correlation between intergenic transcription across PcG response elements (PREs) at the BX-C locus and the relief of silencing by PcG complexes (Bender and Fitzgerald 2002; Hogga and Karch 2002; Rank et al. 2002). Most recently, studies using a transgenic reporter system have demonstrated that transcription in either direction across a PRE blocks repression by PcG complexes and that this activated state is inheritable (Schmitt et al. 2005). A second example occurs at the 50-kb human $\beta$-globin locus, where developmentally regulated intergenic transcription occurs in three large chromatin subdomains and is required for the proper developmental regulation of the five globin genes within this locus (Gribnau et al. 2000). The domains of intergenic transcription correlate with the regions of DNase I sensitivity, again strongly suggesting that transcription confers chromatin changes.

In contrast to cases in which intergenic transcription activates gene expression, we recently reported a case in $S$. cerevisiae in which intergenic transcription represses gene expression (Martens et al. 2004). Our work identified a noncoding RNA, SRG1, which is transcribed from intergenic DNA, and showed that transcription of SRG1 across the promoter of the adjacent SER3 gene represses $S E R 3$ transcription. We provided evidence for a model in which transcription of SRG1 represses SER3 expression by transcription interference. However, the role of $S R G 1$ in normal SER3 regulation was not addressed.

In this work, we use the $S$. cerevisiae SRG1/SER3 system to provide new insights into the physiological role in regulation by an intergenic transcript. First, we show that SRG1 transcription is induced by high serine levels, resulting in repression of SER3. Second, we identify the serine-responsive activator Cha4 as acting directly in SRG1 induction, thereby indirectly repressing SER3. Third, we show that Cha4 recruits the SAGA and Swi/ Snf coactivator complexes to the SRG1 promoter in a serine-dependent manner. Finally, we show that both SAGA and Swi/Snf are required for SER3 repression by facilitating Cha4-dependent activation of SRG1. Taken together, our results show that a physiological response to serine, repression of SER3 transcription, occurs by activating SRG1 intergenic transcription. Based on these results and previous work that implicates Cha4 as a direct activator of the serine catabolism CHA1 gene (Holmberg and Schjerling 1996; Sabet et al. 2003), intergenic transcription provides a mechanism for a single protein to simultaneously activate and repress opposing pathways.

\section{Results}

Serine-dependent regulation of SER3 requires the expression of SRG1 from intergenic DNA

We have previously shown that transcription of SER3 is strongly repressed in YPD, a serine-containing medium, by transcription of SRG1 from intergenic DNA (Fig. 1A; Martens et al. 2004) To investigate a physiological role for this repression mechanism, we performed Northern analysis to assay the response of SER3 and SRG1 RNA levels to changes in serine levels. First, when wild-type cells were grown in minimal medium with serine $(\mathrm{SD}+\mathrm{ser})$ and then shifted to minimal medium without serine $(\mathrm{SD})$, there was a rapid but transient increase in SER3 mRNA levels (Fig. 1B, lanes 1-9). Conversely, SRG1 RNA levels, initially high in the presence of serine, underwent a rapid but transient decrease when the cells were shifted from SD + ser to SD medium. When the complementary shift was performed, from SD to SD + ser medium, the opposite effect was observed, as SER3 mRNA levels decreased, while SRG1 RNA levels were induced to a significantly greater level (Fig. 1C, lanes 1-9|. These results demonstrate that expression of SER3 and SRG1 are tightly but oppositely regulated by the availability of serine.

Next we tested whether transcription of SRG1 is re-

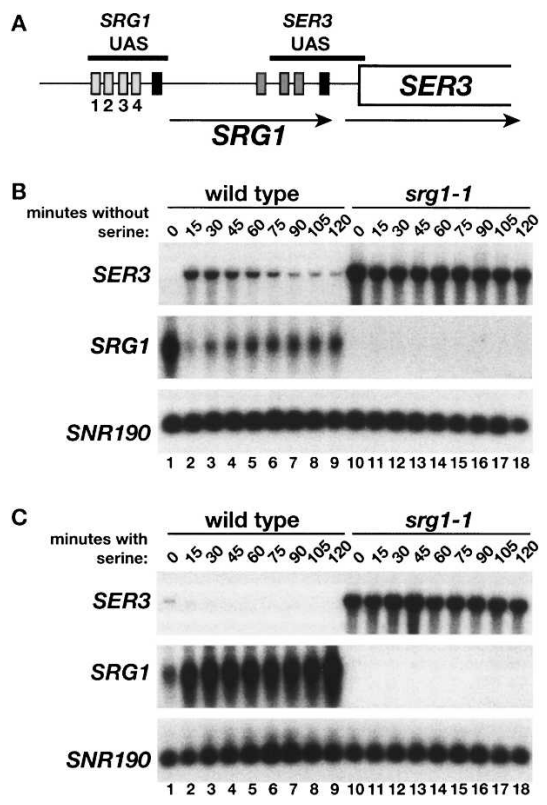

Figure 1. Effect of serine on SER3 and SRG1 expression. (A) A schematic of SRG1 and SER3, showing TATA and putative UAS sequences conserved between $S$. cerevisiae and four related yeast strains. TATA elements are represented by black boxes, the putative SRG1 UAS elements by light-gray boxes $5^{\prime}$ of the SRG1 TATA (labeled 1-4), and the putative SER3 UAS elements by dark-gray boxes $5^{\prime}$ of $S E R 3$ TATA. The horizontal black bars mark the SRG1 and SER3 UAS regions that were amplified by PCR in ChIP experiments described later. The arrows indicate the orientation and positions of the SRG1 and SER3 transcripts. (B) Northern analysis of SER3 and SRG1 was performed on wildtype (FY2472) and $\operatorname{srg} 1-1$ (FY2471; contains a mutation of the SRG1 TATA) strains after a shift from SD + ser to SD medium. SNR190 served as a loading control. Total RNA was isolated at 15-min intervals. These data are representative of three independent experiments. $(C)$ Northern analysis was performed on the strains described in $B$ after the opposite shift, from SD to $\mathrm{SD}+$ ser. These data are representative of three independent experiments. 
quired for the serine-dependent regulation of SER3. To do this we examined RNA levels in an srg1-1 mutant in which mutation of the SRG1 TATA sequence greatly reduces SRG1 RNA levels. In the srg1-1 mutant, SER3 was expressed at high levels independently of the presence or absence of serine (Fig. 1B [lanes 10-18], C [lanes 10-18]). Therefore, serine-dependent regulation of SER3 occurs indirectly through the serine-dependent control of SRG1 transcription.

\section{Cha4 binds to the SRG1 promoter to control serine-dependent transcription of SRG1}

To test for a serine-dependent response element within the SRG1 promoter, we constructed mutations that delete four SRG1 promoter sequences that are highly conserved between $S$. cerevisiae and closely-related yeasts (labeled 1-4 in Fig. 1A; Martens et al. 2004). These mutants were then tested for SRG1 and SER3 mRNA levels in the presence or absence of serine. A deletion that encompasses all four regions (srg1-20) abolished SRG1 expression and caused SER3 derepression in SD + ser medium (Fig. 2A, cf. lanes 1 and 3). Deletion of three of the four individual conserved sequences (UAS1, UAS2, and UAS4) caused no effect on serine-dependent expression of SRG1 or SER3 (Fig. 2A). In contrast, deletion of UAS3 $(\operatorname{srg} 1-23)$ resulted in significant decreases in the induction of SRG1 and repression of SER3 in SD + ser medium (Fig. 2B, cf. lanes 1 and 9).

Further sequence inspection revealed that the UAS3 region overlaps a putative binding site (TGGAGATA CATCTCCA) for Cha4, an S. cerevisiae activator. Cha4 has been previously shown to be a serine-responsive activator of the CHA1 gene, which encodes an enzyme involved in serine catabolism (Holmberg and Schjerling 1996; Sabet et al. 2003). To determine whether Cha4 plays a role in activating SRG1 transcription we constructed and tested three mutations: a deletion of CHA4 $($ cha4s), and both a deletion (srg1-25) and a multiplepoint mutant (srg1-26) of the putative Cha4-binding site. By Northern analysis, we observed greatly decreased levels of SRG1 RNA in all three mutants as compared with a wild-type strain when these strains were grown in SD + ser (Fig. 2B, cf. lanes 3,5,7 and 1). As expected, SER3 repression is also abolished in these three mutants (Fig. 2B).

To confirm a direct role for Cha4 in serine-dependent activation of $S R G 1$, we assayed Cha4 physical association with both the SRG1 and SER3 promoters by chromatin immunoprecipitation (ChIP). Our results show that when cells are grown in SD + ser, Cha4 strongly associates with the UAS region of the SRG1 promoter (Fig. 3A [lanes 9-12], B, top panel); this association is lost in $\operatorname{srg} 1-25$, in which the putative Cha4-binding site in the SRG1 promoter is deleted (Fig. 3A [lanes 17-20], B). We also observed a weak association of Cha4 with the SER3 UAS region (Fig. 3A [lanes 9-12], B). However, this signal is likely caused by the close proximity of the SRG1 and SER3 UAS regions (221 base pairs (bp) between probes), as the SER3 ChIP signal is lost in the srg1-25

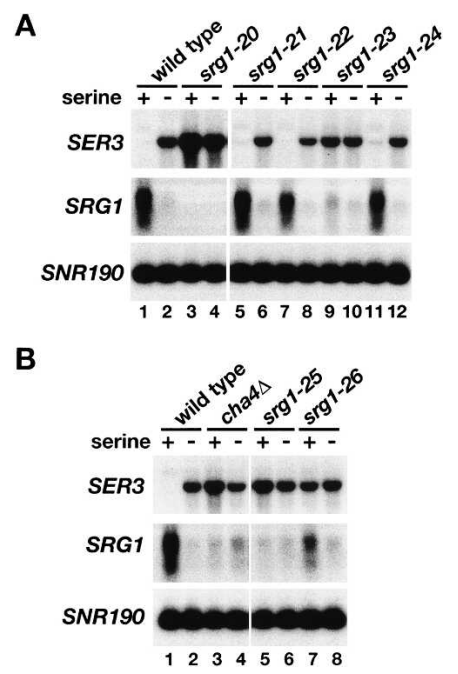

Figure 2. Identification of a serine response element in the SRG1 promoter. (A) Northern analysis of SER3 and SRG1 on a wild-type strain (FY2460) and on a series of srg1 promoter mutants. The srg1-20 mutant (FY2467) has the entire SRG1 UAS region deleted (Fig. 1A, regions 1-4). The srg1-21 (FY2464), srg122 (FY2465), srg1-23 (FY2466), and srg1-24 (FY2461) mutants are deletions of SRG1 UAS sequences 1, 2, 3, and 4, respectively (Fig. 1A). Total RNA was isolated from cells grown in SD + ser medium and from cells that had then been shifted to SD medium for $25 \mathrm{~min}$. SNR190 served as a loading control. These data are representative of three independent experiments. $(B)$ Northern analysis of SER3 and SRG1 was performed on wildtype (FY2460), cha4t (FY2459), srg1-25 (FY2463), and srg1-26 (FY2462) strains that were grown in SD + ser and SD medium as described in $A$. The $\operatorname{srg} 1-25$ mutant is a deletion of a putative Cha4-binding site in the SRG1 promoter and the srg1-26 mutant has a triple-point mutation within this putative Cha4-binding site. These data are representative of three independent experiments.

mutant (Fig. 3A [lanes 17-20], B). Taken together, our results show that Cha4 binds to a single site within the SRG1 UAS to activate SRG1, thereby repressing SER3 transcription in serine-rich medium.

To test if Cha4 binding to SRG1 is serine dependent, we compared Cha4 binding in minimal media with or without serine. Cha4 binding was only mildly decreased after shifting cells to SD medium for 25 min (Fig. 3A [lanes 13-16], B [bottom panel]), a condition in which $S R G 1$ transcription is at a low level (Fig. 1B). This result suggests that Cha4 binding is not strongly controlled by serine levels; rather, induction of SRG1 in response to serine involves an event subsequent to Cha4 binding. This finding is consistent with previous studies that have characterized Cha4 activation at CHA1 (Sabet et al. 2003).

The Spt3 and Spt8 subunits of SAGA are required to repress SER3

Whole-genome expression analyses originally highlighted SER3 as a gene with interesting regulation, as 
A

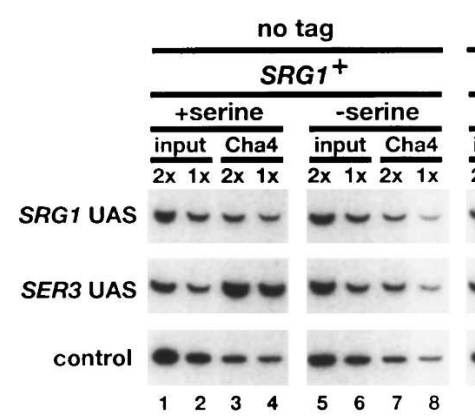

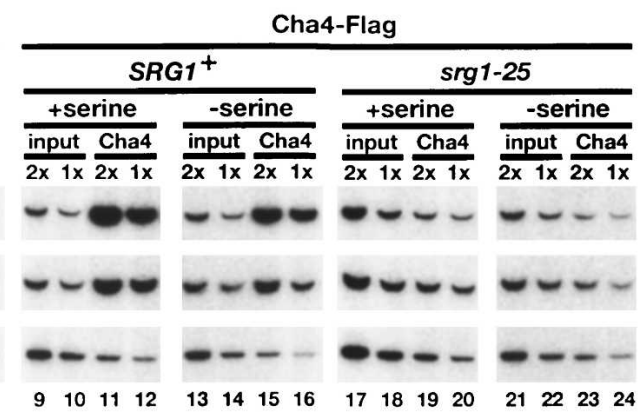

B

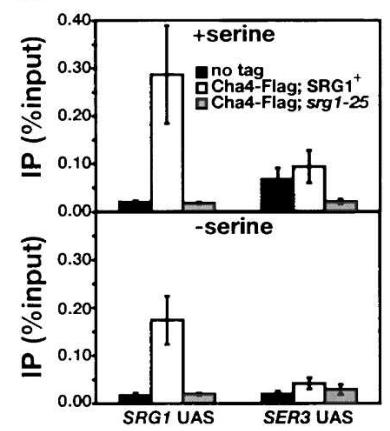

Figure 3. ChIP analysis of Cha4 association with the SRG1 promoter. (A) ChIP analysis of Cha4 was performed on wild-type (FY2470) and $\operatorname{srg} 1-25$ (FY2468) strains expressing Cha4-Flag and on an untagged control strain (FY1350). Cha4-Flag was immunoprecipitated with anti-Flag antibody from cells grown in SD + ser medium (+serine) and from cells that had been shifted from SD + ser to SD medium for $25 \mathrm{~min}$ (-serine). A representative set of PCR reactions that amplify the SRG1 UAS and SER3 UAS regions (see diagram in Fig. 1A) from twofold dilutions of chromatin is shown. The control primer set amplifies a region of chromosome V that lacks open reading frames (Komarnitsky et al. 2000). (B) Quantitation of ChIP analysis. The amount of SRG1 UAS or SER3 UAS that was amplified from immunoprecipitated DNA is expressed as a percentage of the amount of input DNA. Each bar represents the average and standard error from three independent experiments.

those studies suggested that both Swi/Snf (Holstege et al. 1998; Sudarsanam et al. 2000) and SAGA (Holstege et al. 1998) serve as repressors of SER3. Our more recent studies have examined the role of Swi/Snf in SER3 repression (Martens and Winston 2002; Martens et al. 2004). Now, to further investigate the role of SAGA in SER3 repression, we measured SER3 mRNA levels in eleven mutants, each lacking a different SAGA subunit and impairing different SAGA actitivies. Our results show that only particular classes of SAGA components are required for SER3 repression. Two SAGA mutants believed to impair TBP recruitment, spt $3 \Delta$ and spt $8 \Delta$, both had dramatically increased levels of SER3 mRNA, similar to that previously observed for a deletion of $S N F 2$, which encodes the catalytic subunit of the Swi/Snf chromatin remodeling complex (Fig. 4A, lanes 1-4). SER3 mRNA levels were also greatly derepressed in three other SAGA mutants, ada1 $\Delta$, spt20 $\Delta$, and spt $7 \Delta$ although not quite to the extent observed for spt $3 \Delta$ and spt $8 \Delta$ (Fig. 4A, lanes 5-7). Ada1, Spt20, and Spt7 are required for the structural integrity of SAGA (Grant et al. 1997; Sterner et al. 1999). In contrast, none of the other SAGA mutants tested, including gcn $5 \Delta$ and ubp $8 \Delta$, had any effect on SER3 repression (Fig. 4A, lanes 8-11). These results strongly suggest that the Spt3/Spt8 TBP recruitment activity of SAGA (Dudley et al. 1999b; Warfield et al. 2004) is required for SER3 repression, while the Gcn5 histone acetyltransferase activity (Grant et al. 1997) and the Ubp8 deubiquitylation activity (Henry et al. 2003; Daniel et al. 2004) of SAGA play little, if any, role.

To address the roles of both SAGA and Swi/Snf in activating $S R G 1$ transcription, we assayed SRG1 RNA levels in both spt $3 \Delta$ and snf2 $\Delta$ mutants. To look under conditions maximally inducing for $S R G 1$, cells were grown in $\mathrm{SD}+$ ser medium. Northern analysis shows that the serine-induced levels of SRG1 were decreased, albeit modestly, in both mutants compared with wild type. In the snf2 $\Delta$ mutant, induction of $S R G 1$ was re- duced 1.5-fold to twofold (Fig. 4B, lanes 2,3) consistent with both our previous Northern analysis (Martens et al. 2004) and with ChIP experiments in which we found a twofold decrease in the association of the Rpb3 subunit of RNA polymerase II to $S R G 1$ in a snf2 $\Delta$ strain (data not shown). In the spt3s mutant, induction was reduced two- to threefold (Fig. 4B, lanes 4,5). Therefore, our results show that both SAGA and Swi/Snf are required for full induction of $S R G 1$ in serine-rich medium.

\section{Association of SAGA and Swi/Snf to the SRG1 promoter is dependent on Chat and serine}

Our results show that Cha4, Spt3, and Snf2 are required for full induction of $S R G 1$ in medium with serine, suggesting that Cha4 recruits SAGA and Swi/Snf to the SRG1 UAS. To test this possibility, we performed ChIP experiments on Swi2 of Swi/Snf (Fig. 5A) and Adal of SAGA (Fig. 5B). In wild-type strains, we detected a strong association of both proteins with the SRG1 UAS, with a lower level of association over the adjacent SER3 UAS (Fig. 5, top panels). Significantly, the association of both Snf2 and Adal with these regions was reduced to background levels when either CHA4 (cha4D) or the Cha4binding site in the SRG1 UAS (srg1-25) was deleted. These results strongly suggest that both Swi/Snf and SAGA are recruited to the SRG1 UAS by Cha4.

Serine-dependent activation by Cha4 appears to involve a step that occurs subsequent to Cha4 binding (Fig. 3B; Sabet et al. 2003; our results). To test whether recruitment of SAGA and Swi/Snf by Cha 4 are affected by serine levels, we examined the association of Snf2 and Adal to the SRG1 UAS after shifting cells from SD + ser to SD medium. In contrast to Cha4, which remained associated with the SRG1 UAS in the absence of serine, there was no significant recruitment of either Snf2 or Adal when cells were grown in SD medium (Fig. 5A,B; 
A
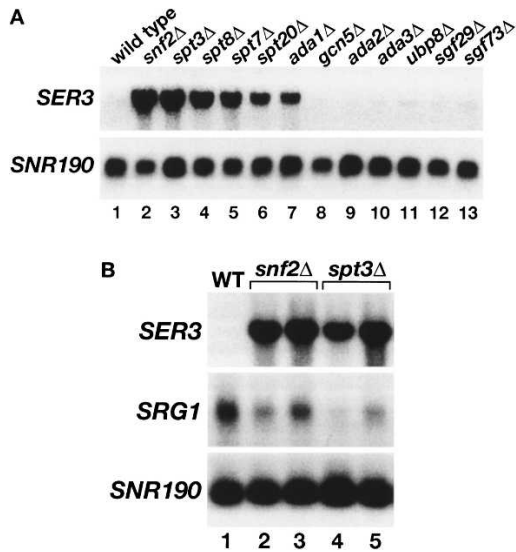

Figure 4. Repression of $S E R 3$ is dependent on the Spt3 and Spt8 subunits of SAGA. (A) Northern analysis of SER3. Total RNA was isolated from wild-type (FY3), snf2 $\Delta$ (FY1360), spt3 $\Delta$

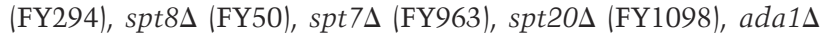

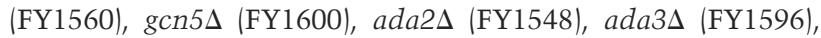
ubp8s (FY2473), sgf29s (FY2474), and sgf73s (FY2475) strains that were grown in YPD. SNR190 served as a loading control. These data are representative of three independent experiments. (B) Northern analysis of SER3 and SRG1. Total RNA was isolated from wild-type (FY4), two snf2 (FY2150 and FY2151), and two spt3s (FY930 and FY2142) strains that were grown in $\mathrm{SD}+$ ser medium. RNA levels were averaged for at least three independent experiments. SER3 mRNA levels are $31.4 \pm 2.7$ and $27.5 \pm 2.3$ in snf $2 \Delta$ and spt $3 \Delta$ strains, respectively, as compared with wild type. SRG1 RNA levels are $0.59 \pm 0.06$ and $0.37 \pm 0.07$ in snf $2 \Delta$ and spt $3 \Delta$ strains, respectively, as compared with wild type.

bottom panels). Therefore, Cha4 recruits SAGA and Swi/ Snf to the SRG1 UAS in a serine-dependent manner.

\section{Both SAGA and Swi/Snf are required for SRG1-mediated transcription interference}

Because snf2 $2 \Delta$ and spt $3 \Delta$ have only modest defects in $S R G 1$ transcription we performed experiments to determine the effects of these mutations on transcription interference by SRG1. Since the direct activators of SER3 have not been identified, we integrated the $S R G 1$ promoter at the GAL1 locus, 5' of the GAL1 UAS (Fig. 6A), in wild-type, snf2 $\Delta$, and spt $3 \Delta$ strains. These strains were analyzed for both transcription from the SRG1 promoter and Gal4 binding. (Note that in this experiment GAL1 will not be transcribed as the cells are grown in glucose, a condition permissive for Gal4 binding, but repressive for GAL1 transcription. These conditions were necessary to avoid secondary effects of snf2 $\Delta$ and spt3s mutations on galactose-grown cells.) Using a probe to the GAL1 UAS region we detected transcription from the SRG1 promoter that appears to extend to the end of GAL1. Consistent with our previous results, transcription from the $S R G 1$ promoter is decreased two- to threefold in snf2s, and spt3s mutants and is undetectable in an srg1-1 mutant, containing a mutated SRG1 TATA (Fig. 6B; Martens et al. 2004). ChIP analysis demonstrates that, like the $\operatorname{srg} 1-1$ mutant, snf2 $\Delta$ and spt $3 \Delta$ mu- tations relieve the inhibition of Gal4 binding observed with wild type SRG1 (Fig. 6C). Unexpectedly, Gal4 binding to the GAL1 UAS is three- to fourfold higher in the snf2 $\Delta$ strain than in either the spt3s or gal1::srg1-1p strains (Fig. 6C). However, in a control experiment, we found that Gal4 binding to wild-type GAL1 was similarly increased in a snf2 $\Delta$ strain (Supplementary Fig. S2), which would account for this difference. The requirement for Swi/Snf and SAGA in transcription interference with Gal4 binding in this construct strongly suggests that Swi/Snf and SAGA are required for transcription interference of SER3. Although we cannot rule out the possibility that Swi/Snf and/or SAGA have additional functions in SER3 repression, our results suggest that one function of SAGA and Swi/Snf in SER3 repression is to facilitate transcription interference by activating transcription of SRG1.

\section{Discussion}

In this work we have elucidated a physiological role for an intergenic transcript in gene regulation. Our results have shown that the repression of the S. cerevisiae SER3 gene in response to high levels of serine occurs by the induction of intergenic transcription of SRG1. Our results also show that the serine-dependent induction of
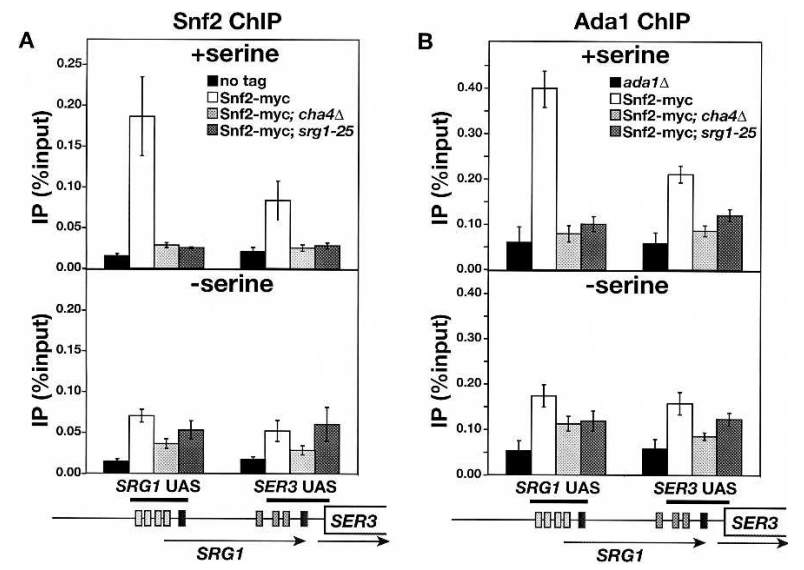

Figure 5. ChIP analysis of Snf2 and Ada1 association to the SRG1 promoter. (A) ChIP analysis of Snf2 was performed on wild-type (FY2470), cha4s (FY2469), and srg1-25 (FY2468) strains expressing Snf2-myc and an untagged control strain (FY1350). Snf2-myc was immunoprecipitated with anti-myc A14 antibody from chromatin isolated from cells that were grown in SD + ser medium (+serine) and from cells that were shifted from $\mathrm{SD}+$ ser to SD medium for $25 \mathrm{~min}$ (-serine). The amounts of SRG1 UAS and SER3 UAS that were amplified from immunoprecipitated DNA are expressed as percentages of the amounts of input DNA. Each bar represents the average and standard error from three independent experiments. (B) ChIP analysis of Ada1. Ada1 was immunoprecipitated with anti-Ada1 antibody from the same chromatin that is described in $A$ with the exception of the untagged control strain, which was replaced by an ada1s control strain (FY1560). Each bar represents the average amount and standard error of three independent experiments. 
Martens et al.
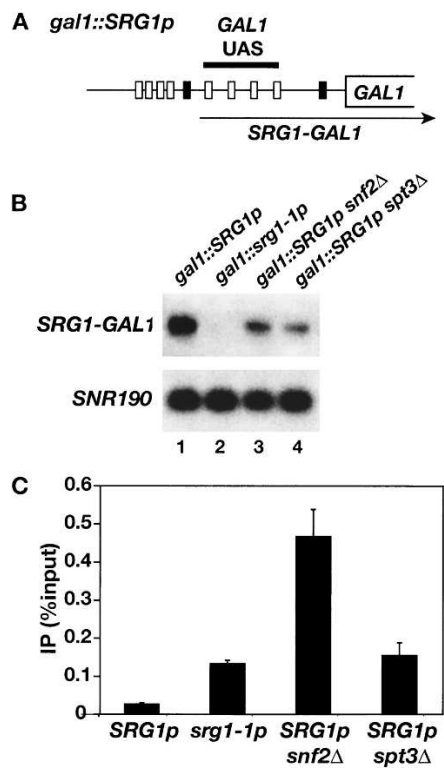

Figure 6. Effect of $s n f 2 \Delta$ and $s p t 3 \Delta$ on transcription interference by $S R G 1$. (A) A schematic of gal1::SRG1p. The SRG1 UAS (gray boxes) and TATA (left-most black box) sequences were integrated into the GAL1 promoter, 5' of the four Gal4-binding sites (white boxes). The arrow indicates the srg1-GAL1 RNA that is transcribed as a result of the SRG1 promoter insertion. The gal1::srg1-1p allele contains a similar insertion of the srg1-1 TATA mutant promoter. Under the conditions of the experiment, with cells grown on glucose, there is no transcription from the normal GAL1 initiation site; however, Gal4 is still bound under these growth conditions (Dudley et al. 1999b; Ren et al. 2000). (B) Northern analysis was performed on gal1::SRG1p (FY2476), gal1::srg1-1p (FY2477), snf2 gal1::SRG1p (FY2478), and spt3s gal1::SRG1p (FY2479) strains that were grown in YPD medium. Transcription from the $S R G 1$ promoter was detected using a probe to the GAL1 UAS (SRG1GAL1). SNR190 RNA was measured as a loading control. (C) ChIP analysis of Gal4 was performed on the same strains described in $B$. The amount of GAL1 UAS that was PCR-amplified from immunoprecipitated DNA was calculated relative to the amount amplified from input DNA. Each bar represents the average and standard error from three experiments.

SRG1 depends upon Cha4, which binds to the SRG1 promoter, and that activation by Cha4 requires the SAGA and Swi/Snf coactivator complexes.

Taken together with previous studies (Holmberg and Schjerling 1996; Sabet et al. 2003), our results suggest a model in which the control of $S R G 1$ intergenic transcription is the primary mechanism that regulates SER3 in response to changes in serine. In this model, $S R G 1$ transcription also allows Cha4 to serve as both an activator and repressor of transcription. When cells are exposed to high levels of serine, Cha4 directly activates the CHA1 gene, required for serine catabolism, and indirectly represses the SER3 gene via activation of SRG1 (Fig. 7, top panel). The requirements for CHA1 activation may be similar to those for SRG1, as CHA1 activation is impaired in both snf $2 \Delta$ and spt $3 \Delta$ mutants, albeit only modestly in snf2 $\Delta$, and both Swi/Snf and SAGA are recruited to the CHA1 promoter in a serine-dependent fashion (Supplementary Fig. S1; R. Morse, pers. comm.). In serine-starvation conditions, the opposite regulation occurs, as Cha4 no longer recruits SAGA and Swi/Snf, resulting in a reversal of the transcription states of CHA1 and SER3 (Fig. 7, bottom panel; Supplementary Fig. S1). Thus, $S$. cerevisiae can quickly adapt to changes in intracellular serine by coordinately regulating serine catabolism and biosynthesis using a single activator. This model also illustrates a mechanism by which intergenic transcription increases the potential functions of regulatory proteins as it allows activators to act indirectly as repressors.

In addition to the factors identified in this work that control SRG1 transcription in response to serine levels, other physiological conditions and factors may play important roles in this regulatory system. Sequence conservation suggests that, in addition to the Cha4-binding site, other $S R G 1$ promoter sequences may be required for SRG1 transcription under some conditions (Fig. 2A; Cliften et al. 2003; Kellis et al. 2003; Martens et al. 2004). These sequences may respond to other physiological signals that control serine levels, such as general nitrogen metabolism, one-carbon metabolism, and fatty acid biosynthesis. In addition, we have previously identified a region of the SER3 promoter that is required for SER3 activation (Martens et al. 2004); however, the factors that bind to this putative SER3 UAS remain unknown and we have not found any evidence for a role for this region in response to serine. We also note that histone $\mathrm{H} 3$ mutants that cause defects in SER3 repression have been identified (Sabet et al. 2003; Duina and Winston 2004). The analysis of these mutants, as well as the identification of other factors required for SRG1 and SER3 regulation, will provide further insight into the physiological relevance for regulation of SER3 by intergenic transcription.

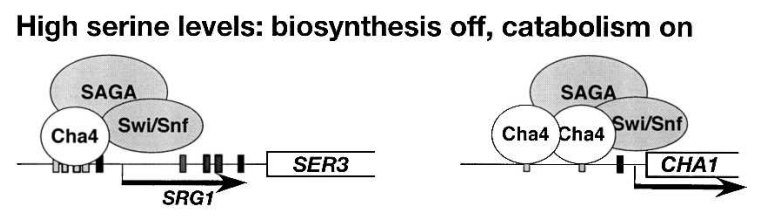

Serine starvation: biosynthesis on, catabolism off

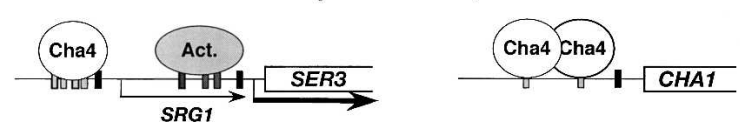

Figure 7. A model for the coordinated regulation of serine biosynthesis and catabolism by Cha4. (Top) In the presence of high serine levels, Cha4 indirectly represses the serine biosynthetic gene SER3 via activation of SRG1 and directly activates the serine catabolic gene CHA1. (Bottome) Under serine-starvation conditions, when Cha4 is no longer able to recruit SAGA and Swi/Snf, the expression states of SER3 and CHA1 are reversed. In this model, the expression of SER3 also requires putative activators (Act.) that bind to the previously identified SER3 UAS (Martens et al. 2004). Thus, Cha4 can act as both an activator and as a repressor in response to serine. 
Another aspect of the SER3/SRG1 regulatory system that remains to be determined is the role of serine in the Cha4-dependent recruitment of SAGA and Swi/Snf. Like many $S$. cerevisiae activators that respond to intracellular metabolites (for review, see Sellick and Reece 2005), Cha4 binding to DNA is not significantly affected by serine levels (our results; Sabet et al. 2003). Possibly, serine or an intermediate in the serine biosynthetic pathway directly modulates Cha4 activity by inducing a conformational change that facilitates recruitment of SAGA and Swi/Snf. A similar mechanism has been suggested for Put3, an activator of the proline-utilization pathway, as proline directly interacts with Put 3 to cause conformational changes that induce the transcription activity of Put3 (Axelrod et al. 1991; Sellick and Reece 2003). Alternatively, serine may indirectly control Cha4 activity by controlling the interaction of Cha4 with an as-yetunidentified second protein, similar to the galactose-mediated regulation of Gal4 by interaction with Gal80 (Sellick and Reece 2005).

A number of different mechanisms have been proposed for transcription interference (for review, see Shearwin et al. 2005) and other naturally occurring cases of transcription interference have been identified (for examples, see Hausler and Somerville 1979; Cullen et al. 1984; Proudfoot 1986; Corbin and Maniatis 1989; Boussadia et al. 1997; Moseley et al. 2002). Our data show that loss of either SAGA or Swi/Snf causes only a two- to threefold decrease in SRG1 RNA levels, yet this modest decrease appears to be sufficient to abolish interference as it is accompanied by a 50 -fold increase in SER3 mRNA levels. One possibility is that there is a threshold level of SRG1 transcription that is sufficient to confer transcription interference and that a modest decrease in that level allows activators full access to the SER3 promoter. However, given the modest effects on SRG1 RNA levels observed in the $s n f 2 \Delta$ and $s p t 3 \Delta$ mutants, SAGA and Swi/Snf may play additional roles to repress SER3 besides SRG1 activation. Consistent with this possibility, Snf2-dependent changes in chromatin structure extend to the SER3 TATA sequence (Martens and Winston 2002). Therefore, there may be two roles for Swi/Snf in $S E R 3$ repression, through activation of SRG1 and by inhibition of factor binding to the SER3 promoter.

In conclusion, our work has demonstrated a mechanism by which the regulation of noncoding, intergenic transcription provides a physiologically important response in a metabolic signaling pathway. Studies in other systems, including the Drosophila BX-C complex and the human $\beta$-globin locus, have demonstrated other cases where regulation of noncoding, intergenic transcription plays important regulatory roles (Gribnau et al. 2000; Schmitt et al. 2005). Additional recent studies have shown that noncoding transcription is widespread (Mattick 2003; Morey and Avner 2004; Huttenhofer et al. 2005; Johnson et al. 2005) and that a significant fraction is regulated (Cawley et al. 2004; Kim et al. 2005). Taken together, these studies suggest that the control of noncoding, intergenic transcription may represent a broadly used mechanism for transcriptional regulation.

\section{Materials and methods}

\section{S. cerevisiae strains and media}

All S. cerevisiae strains used (Table 1) are isogenic with a $G A L 2^{+}$derivative of S288C (Winston et al. 1995). Strains were constructed by standard methods, either by crosses or by transformation (Ausubel et al. 1991). Details are available upon request. The spt8A302::LEU2 (Eisenmann et al. 1994), spt3-202 (Winston and Minehart 1986), spt3A203::TRP1 (Happel and Winston 1992), spt7 402::LEU2 (Gansheroff et al. 1995), spt20A100::URA3 (Roberts and Winston 1996), snf2 $::$ LEU2 (Cairns et al. 1996), snf2 $1::$ HIS3 (Abrams et al. 1986),

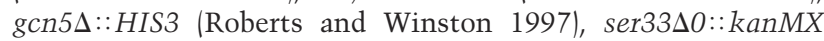
(Martens and Winston 2002), srg1-1 (Martens et al. 2004), RPB3-HA::LEU2 (Kolodziej et al. 1990), SNF2-C18myc::TRP1 (Martens and Winston 2002), and cha4t0::kanMX (Open Biosystems) alleles have been previously described. The ada2s::HIS3, ada1s::HIS3, and ada3s::HIS3 mutations were constructed by replacing their open reading frames with HIS3 (S. Roberts and F. Winston, unpubl.). The ubp8s0::kanMX, $s g f 29 \Delta 0:: k a n M X$, and $s g f 73 \Delta 0:$ kanMX mutations were constructed by replacing their open reading frames with the kanMX marker (Brachmann et al. 1998). The srg1-20 mutation replaces SRG1 promoter sequences from -660 to -560 (relative to the SER3 ATG) with three copies of the myc epitope. The srg1-21, srg1-22, srg1-23, srg1-24, and $\operatorname{srg} 1-25$ mutations replace SRG1 promoter sequences -659 to $-646,-644$ to $-636,-611$ to -604 , -590 to -582 , and -612 to -592 each with an AvrII site (CCTAGG). The $\operatorname{srg} 1-26$ mutation contains three point mutations in the Cha4 consensus binding site within the SRG1 promoter (TGGAGATACATCTCCA to ctGAGATACATCTCaA). This mutant sequence has been shown to be defective for Cha4 binding (Holmberg and Schjerling 1996). The CHA4Flag::kanMX allele encodes one copy of the Flag epitope at the $3^{\prime}$ end of CHA4, which was constructed by PCR-mediated integration using plasmid pDM714 (kind gift from D. Moazed, Harvard Medical School, Boston, MA). The gal1::SRG1p allele contains the SRG1 promoter and transcription initiation sites (base pairs -713 to -445 relative to the SER3 ATG) at position -556 of GAL1 (relative to GAL1 ATG). The gal1::srg1-1p allele has an insertion of the same DNA except with the srg1-1 promoter containing the TATA mutation. As indicated, strains were grown in either YPD ( $1 \%$ yeast extract, $2 \%$ peptone, and $2 \%$ glucose $)$ or minimal media $(0.145 \%$ yeast nitrogen base, $0.5 \%$ ammonium sulfate, $2 \%$ glucose, and supplemented with required amino acids) with $1 \mathrm{mM}$ serine (SD + ser) or without serine (SD).

\section{Northern analysis}

Northern hybridization analysis was performed as previously described (Ausubel et al. 1991). Probes for SER3, SRG1, and SNR190 were previously described (Martens and Winston 2002; Duina and Winston 2004; Martens et al. 2004). A probe specific to the GAL1 5' UTR was generated by random labeling a PCR product, containing the GAL1 sequence from -443 to -157 (relative to the GAL1 ATG), amplified from genomic DNA. RNA levels were quantitated using a PhosphorImager (Molecular Dynamics) and normalized to SNR190, whose levels are unaffected by the mutations and growth conditions studied here.

\section{ChIP analysis}

ChIP analysis was performed as previously described (Dudley et al. 1999b; Martens and Winston 2002) with the following modifications: For the Cha4, Snf2, and Ada1 ChIPs, 200-mL cultures 
Martens et al.

Table 1. Saccharomyces cerevisiae strains

\begin{tabular}{|c|c|}
\hline Name & Genotype \\
\hline FY3 & MATa ura3-52 \\
\hline FY4 & MATa (prototroph) \\
\hline FY50 & 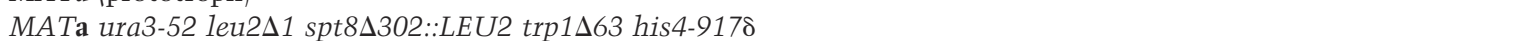 \\
\hline FY294 & 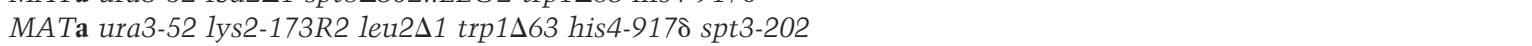 \\
\hline FY930 & MATa ura3-52 lys2-173R2 his3د200 spt3-202 \\
\hline FY963 & 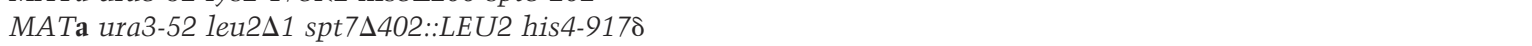 \\
\hline FY1098 & 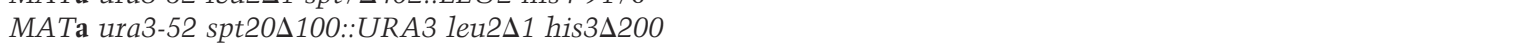 \\
\hline FY1350 & 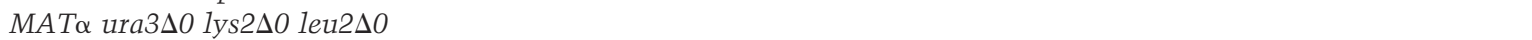 \\
\hline FY1360 & 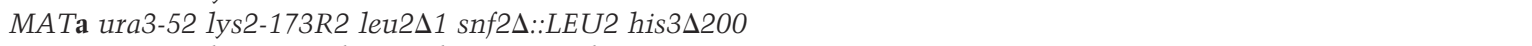 \\
\hline FY1548 & 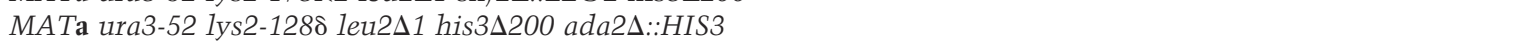 \\
\hline FY1560 & 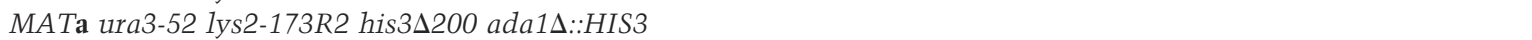 \\
\hline FY1596 & 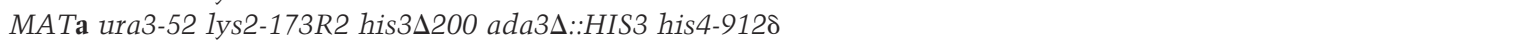 \\
\hline FY1600 & 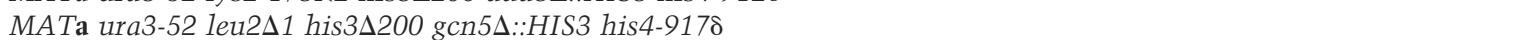 \\
\hline FY2142 & MAT $\alpha$ spt $3 \Delta 0::$ kanMX \\
\hline FY2150 & MATa snf2 $\Delta 0::$ kanMX \\
\hline FY2151 & MATa snf2 $0:: \operatorname{kan} M X$ \\
\hline FY2245 & 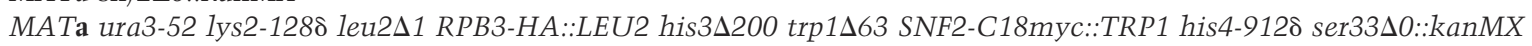 \\
\hline FY2459 & 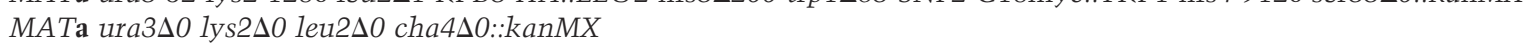 \\
\hline FY2460 & 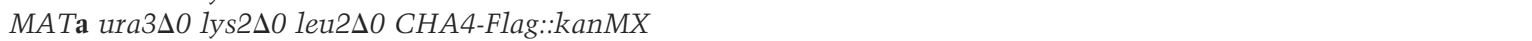 \\
\hline FY2461 & 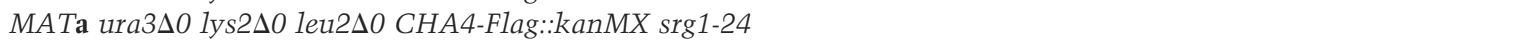 \\
\hline FY2462 & 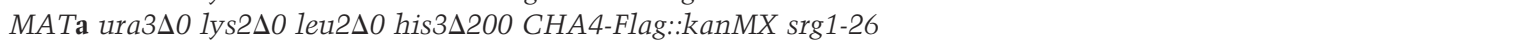 \\
\hline FY2463 & 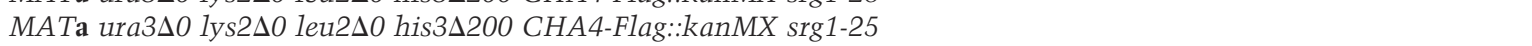 \\
\hline FY2464 & 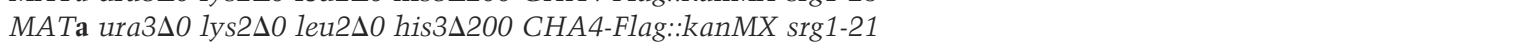 \\
\hline FY2465 & 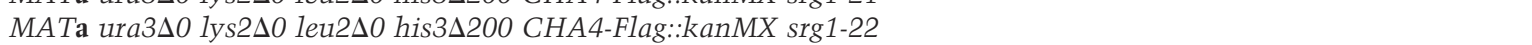 \\
\hline FY2466 & 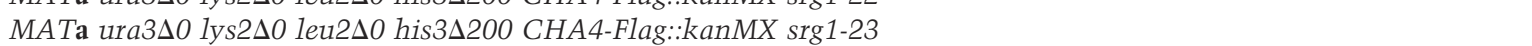 \\
\hline FY2467 & 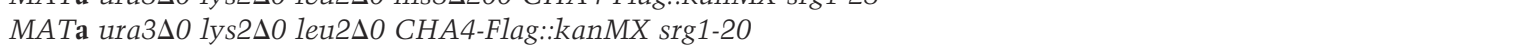 \\
\hline FY2468 & 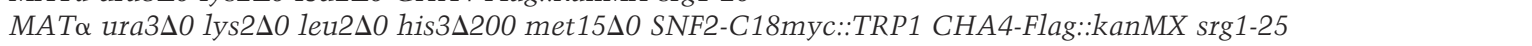 \\
\hline FY2469 & 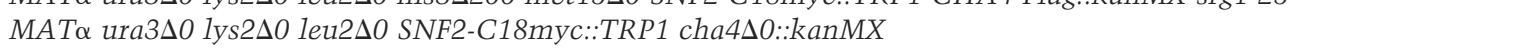 \\
\hline FY2470 & 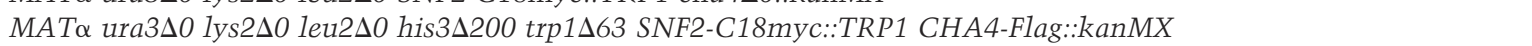 \\
\hline FY2471 & 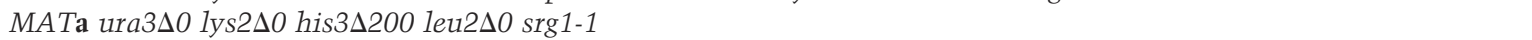 \\
\hline FY2472 & 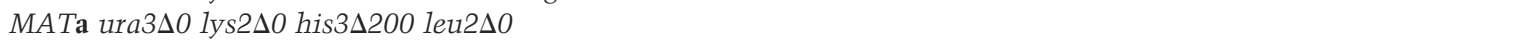 \\
\hline FY2473 & 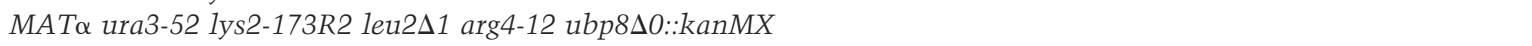 \\
\hline FY2474 & 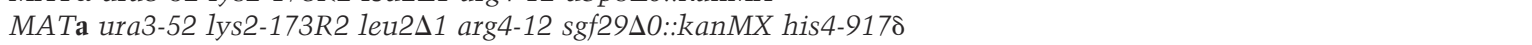 \\
\hline FY2475 & 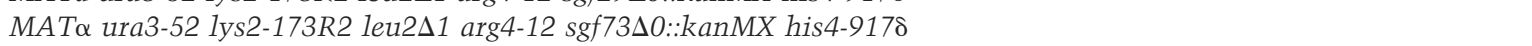 \\
\hline FY2476 & 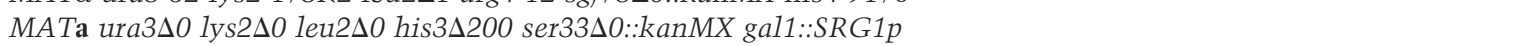 \\
\hline FY2477 & 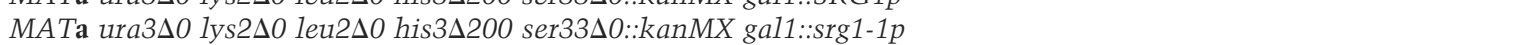 \\
\hline FY2478 & 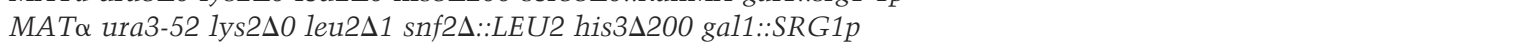 \\
\hline FY2479 & 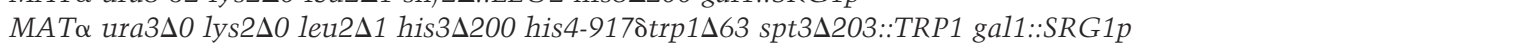 \\
\hline FY2502 & 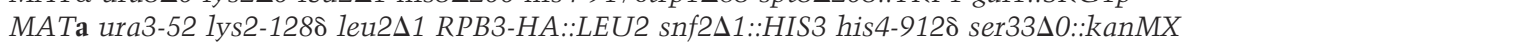 \\
\hline
\end{tabular}

were grown to a density of $0.8 \times 10^{7}$ to $1.0 \times 10^{7}$ cells $/ \mathrm{mL}$ in $\mathrm{SD}+\mathrm{ser}$ medium and then split into two equal volumes. One half was treated with buffered formaldehyde $(11 \%$ formaldehyde, $0.1 \mathrm{M} \mathrm{NaCl}, 1 \mathrm{mM}$ EDTA, and $50 \mathrm{mM}$ Hepes- $\mathrm{KOH}$ at $\mathrm{pH}$ $7.5)$ to a final concentration of $1 \%$. Cells from the other half of the culture were harvested by centrifugation, resuspended in SD medium, incubated for $25 \mathrm{~min}$ at $30^{\circ} \mathrm{C}$, and then treated with buffered formaldehyde to a final concentration of $1 \%$. The following antibodies were used for ChIP from one-fifth of the total amount of cross-linked chromatin: $4 \mu \mathrm{L}$ of mouse A14 anti-myc ascites (Santa Cruz Biotechnology), $2 \mu \mathrm{L}$ of rabbit anti-Flag M2 serum (Sigma-Aldrich, Inc), and $1 \mu \mathrm{L}$ of rabbit anti-Adal serum (a generous gift from L. Guarente, Massachusetts Institute of Technology, Cambridge, MA). Input DNA $(0.002 \%$ and $0.001 \%)$ and immunoprecipitated DNA $(4 \%$ and $2 \%$ for Cha4-Flag and Snf2-myc, $2 \%$ and $1 \%$ for Ada1) were subjected to quantitative radioactive PCR and the products were separated on an $8 \%$ nondenaturing polyacrylamide gel. For the Gal4 ChIPs, 200-mL cultures were grown to a density of $1 \times 10^{7}$ to $2 \times 10^{7}$ cells $/ \mathrm{mL}$ in YPD and then $37 \%$ formaldehyde was added to a final concentration of $1 \%$. Gal4 was immunoprecipitated from one-tenth of the total amount of cross-linked chromatin with $1 \mu \mathrm{L}$; of anti-Gal4 serum (Santa Cruz Biotechnology, Inc.). Input DNA $(0.002 \%$ and $0.001 \%)$ and immunoprecipitated DNA $(1.33 \%$ and $0.67 \%$ ) were analyzed as described for the Cha4 ChIPs. The PCR primers amplify the following regions whose coordinates are given relative to the SER3 ATG: SRG1 UAS primers amplify a 259-bp product from -714 to -456 and SER3 UAS primers amplify a 298 -bp product from -234 to +65 . Primers that amplify the GAL1 UAS and the control region from chromosome V that lacks open reading frames have been previously described (Dudley et al. 1999a; Komarnitsky et al. 2000). All ChIP experiments were quantitated by PhosphorImager analysis (Molecular Dynamics). Association of factors to specific DNA sequences was calculated as a percentage of the amount of coimmunoprecipitated DNA relative to the input DNA.

\section{Acknowledgments}

We thank Dominique Helmlinger and Natalie Kuldell for valuable comments on the manuscript and Amine Nourani and 
Randy Morse for helpful discussions. This work was supported by NIH grant GM32967 to F.W., by a postdoctoral fellowship from the Canadian Institute for Health Research to J.A.M, and by a predoctoral fellowship from the Howard Hughes Medical Institute to P.-Y.J.W.

\section{References}

Abrams, E., Neigeborn, L., and Carlson, M. 1986. Molecular analysis of SNF2 and SNF5, genes required for expression of glucose-repressible genes in Saccharomyces cerevisiae. Mol. Cell. Biol. 6: 3643-3651.

Andersen, A.A. and Panning, B. 2003. Epigenetic gene regulation by noncoding RNAs. Curr. Opin. Cell Biol. 15: 504.

Ausubel, F.M., Brent, R., Kingston, R.E., Moore, D.D., Seidman, J.G., Smith, J.A., and Struhl, K.E. 1991. Current protocols in molecular biology. Greene Publishing Associates and WileyInterscience, New York.

Axelrod, J.D., Majors, J., and Brandriss, M.C. 1991. Proline-independent binding of PUT3 transcriptional activator protein detected by footprinting in vivo. Mol. Cell. Biol. 11: 564567.

Bender, W. and Fitzgerald, D.P. 2002. Transcription activates repressed domains in the Drosophila bithorax complex. Development 129: 4923-4930.

Bernstein, E. and Allis, C.D. 2005. RNA meets chromatin. Genes \& Dev. 19: 1635-1655.

Bertone, P., Stolc, V., Royce, T.E., Rozowsky, J.S., Urban, A.E., Zhu, X., Rinn, J.L., Tongprasit, W., Samanta, M., Weissman, S., et al. 2004. Global identification of human transcribed sequences with genome tiling arrays. Science 306: 22422246.

Boussadia, O., Amiot, F., Cases, S., Triqueneaux, G., JacqueminSablon, H., and Dautry, F. 1997. Transcription of unr (upstream of $\mathrm{N}$-ras) down-modulates $\mathrm{N}$-ras expression in vivo. FEBS Lett. 420: 20-24.

Brachmann, C.B., Davies, A., Cost, G.J., Caputo, E., Li, J., Hieter, P., and Boeke, J.D. 1998. Designer deletion strains derived from Saccharomyces cerevisiae S288C: A useful set of strains and plasmids for PCR-mediated gene disruption and other applications. Yeast 14: 115-132.

Cairns, B.R., Levinson, R.S., Yamamoto, K.R., and Kornberg, R.D. 1996. Essential role of Swp73p in the function of yeast Swi/Snf complex. Genes \& Dev. 10: 2131-2144.

Cawley, S., Bekiranov, S., Ng, H.H., Kapranov, P., Sekinger, E.A., Kampa, D., Piccolboni, A., Sementchenko, V., Cheng, J., Williams, A.J., et al. 2004. Unbiased mapping of transcription factor binding sites along human chromosomes 21 and 22 points to widespread regulation of noncoding RNAs. Cell 116: 499-509.

Chen, J., Sun, M., Lee, S., Zhou, G., Rowley, J.D., and Wang, S.M. 2002. Identifying novel transcripts and novel genes in the human genome by using novel SAGE tags. Proc. Natl. Acad. Sci. 99: 12257-12262.

Cheng, J., Kapranov, P., Drenkow, J., Dike, S., Brubaker, S., Patel, S., Long, J., Stern, D., Tammana, H., Helt, G., et al. 2005. Transcriptional maps of 10 human chromosomes at 5-nucleotide resolution. Science 308: 1149-1154.

Cliften, P., Sudarsanam, P., Desikan, A., Fulton, L., Fulton, B., Majors, J., Waterston, R., Cohen, B.A., and Johnston, M. 2003. Finding functional features in Saccharomyces genomes by phylogenetic footprinting. Science 301: 71-76.

Corbin, V. and Maniatis, T. 1989. Role of transcriptional interference in the Drosophila melanogaster Adh promoter switch. Nature 337: 279-282.

Cullen, B.R., Lomedico, P.T., and Ju, G. 1984. Transcriptional interference in avian retroviruses-Implications for the promoter insertion model of leukaemogenesis. Nature 307: 241-245.

Cumberledge, S., Zaratzian, A., and Sakonju, S. 1990. Characterization of two RNAs transcribed from the cis-regulatory region of the abd-A domain within the Drosophila bithorax complex. Proc. Nat1. Acad. Sci. 87: 3259-3263.

Daniel, J.A., Torok, M.S., Sun, Z.W., Schieltz, D., Allis, C.D., Yates III, J.R., and Grant, P.A. 2004. Deubiquitination of histone $\mathrm{H} 2 \mathrm{~B}$ by a yeast acetyltransferase complex regulates transcription. J. Biol. Chem. 279: 1867-1871.

Dudley, A.M., Gansheroff, L.J., and Winston, F. 1999a. Specific components of the SAGA complex are required for Gcn4and Gcr1- mediated activation of the his4-912 $\delta$ promoter in Saccharomyces cerevisiae. Genetics 151: 1365-1378.

Dudley, A.M., Rougeulle, C., and Winston, F. 1999b. The Spt components of SAGA facilitate TBP binding to a promoter at a post-activator-binding step in vivo. Genes \& Dev. 13: 2940-2945.

Duina, A.A. and Winston, F. 2004. Analysis of a mutant histone $\mathrm{H} 3$ that perturbs the association of Swi/Snf with chromatin. Mol. Cell Biol. 24: 561-572.

Eisenmann, D.M., Chapon, C., Roberts, S.M., Dollard, C., and Winston, F. 1994. The Saccharomyces cerevisiae SPT8 gene encodes a very acidic protein that is functionally related to SPT3 and TATA-binding protein. Genetics 137: 647-657.

Gansheroff, L.J., Dollard, C., Tan, P., and Winston, F. 1995. The Saccharomyces cerevisiae SPT7 gene encodes a very acidic protein important for transcription in vivo. Genetics 139: $523-536$.

Gottesman, S. 2004. The small RNA regulators of Escherichia coli: Roles and mechanisms. Annu. Rev. Microbiol. 58: $303-$ 328.

Grant, P.A., Duggan, L., Cote, J., Roberts, S.M., Brownell, J.E., Candau, R., Ohba, R., Owen-Hughes, T., Allis, C.D., Winston, F., et al. 1997. Yeast Gen5 functions in two multisubunit complexes to acetylate nucleosomal histones: Characterization of an Ada complex and the SAGA (Spt/Ada) complex. Genes \& Dev. 11: 1640-1650.

Gribnau, J., Diderich, K., Pruzina, S., Calzolari, R., and Fraser, P. 2000. Intergenic transcription and developmental remodeling of chromatin subdomains in the human $\beta$-globin locus. Mol. Cell 5: 377-386.

Happel, A.M. and Winston, F. 1992. A mutant tRNA affects $\delta$-mediated transcription in Saccharomyces cerevisiae. Genetics 132: 361-374.

Hausler, B. and Somerville, R.L. 1979. Interaction in vivo between strong closely spaced constitutive promoters. J. Mol. Biol. 127: 353-356.

Havilio, M., Levanon, E.Y., Lerman, G., Kupiec, M., and Eisenberg, E. 2005. Evidence for abundant transcription of noncoding regions in the Saccharomyces cerevisiae genome. BMC Genomics 6: 93.

Henry, K.W., Wyce, A., Lo, W.S., Duggan, L.J., Emre, N.C., Kao, C.F., Pillus, L., Shilatifard, A., Osley, M.A., and Berger, S.L. 2003. Transcriptional activation via sequential histone $\mathrm{H} 2 \mathrm{~B}$ ubiquitylation and deubiquitylation, mediated by SAGA-associated Ubp8. Genes \& Dev. 17: 2648-2663.

Hogga, I. and Karch, F. 2002. Transcription through the iab-7 cis-regulatory domain of the bithorax complex interferes with maintenance of Polycomb-mediated silencing. Development 129: 4915-4922.

Holmberg, S. and Schjerling, P. 1996. Cha4p of Saccharomyces cerevisiae activates transcription via serine/threonine response elements. Genetics 144: 467-478.

Holstege, F.C., Jennings, E.G., Wyrick, J.J., Lee, T.I., Hengartner, 
C.J., Green, M.R., Golub, T.R., Lander, E.S., and Young, R.A. 1998. Dissecting the regulatory circuitry of a eukaryotic genome. Cell 95: 717-728.

Hurowitz, E.H. and Brown, P.O. 2003. Genome-wide analysis of mRNA lengths in Saccharomyces cerevisiae. Genome Biol. 5: R2.

Huttenhofer, A., Schattner, P., and Polacek, N. 2005. Non-coding RNAs: Hope or hype? Trends Genet. 21: 289-297.

Johnson, J.M., Edwards, S., Shoemaker, D., and Schadt, E.E. 2005. Dark matter in the genome: Evidence of widespread transcription detected by microarray tiling experiments. Trends Genet. 21: 93-102.

Kampa, D., Cheng, J., Kapranov, P., Yamanaka, M., Brubaker, S., Cawley, S., Drenkow, J., Piccolboni, A., Bekiranov, S., Helt, G., et al. 2004. Novel RNAs identified from an in-depth analysis of the transcriptome of human chromosomes 21 and 22. Genome Res. 14: 331-342.

Kapranov, P., Cawley, S.E., Drenkow, J., Bekiranov, S., Strausberg, R.L., Fodor, S.P., and Gingeras, T.R. 2002. Large-scale transcriptional activity in chromosomes 21 and 22 . Science 296: 916-919.

Kellis, M., Patterson, N., Endrizzi, M., Birren, B., and Lander, E.S. 2003. Sequencing and comparison of yeast species to identify genes and regulatory elements. Nature 423: 241-254.

Kim, T.H., Barrera, L.O., Zheng, M., Qu, C., Singer, M.A., Richmond, T.A., Wu, Y., Green, R.D., and Ren, B. 2005. A highresolution map of active promoters in the human genome. Nature 436: 876-880.

Kolodziej, P.A., Woychik, N., Liao, S.M., and Young, R.A. 1990. RNA polymerase II subunit composition, stoichiometry, and phosphorylation. Mol. Cell. Biol. 10: 1915-1920.

Komarnitsky, P., Cho, E.J., and Buratowski, S. 2000. Different phosphorylated forms of RNA polymerase II and associated mRNA processing factors during transcription. Genes \& Dev. 14: 2452-2460.

Lipshitz, H.D., Peattie, D.A., and Hogness, D.S. 1987. Novel transcripts from the Ultrabithorax domain of the bithorax complex. Genes \& Dev. 1: 307-322.

Martens, J.A. and Winston, F. 2002. Evidence that Swi/Snf directly represses transcription in S. cerevisiae. Genes \& Dev. 16: 2231-2236.

Martens, J.A., Laprade, L., and Winston, F. 2004. Intergenic transcription is required to repress the Saccharomyces cerevisiae SER3 gene. Nature 429: 571-574.

Mattick, J.S. 2003. Challenging the dogma: The hidden layer of non-protein-coding RNAs in complex organisms. Bioessays 25: 930-939.

Meister, G. and Tuschl, T. 2004. Mechanisms of gene silencing by double-stranded RNA. Nature 431: 343-349.

Morey, C. and Avner, P. 2004. Employment opportunities for non-coding RNAs. FEBS Lett. 567: 27-34.

Moseley, J.L., Page, M.D., Alder, N.P., Eriksson, M., Quinn, J., Soto, F., Theg, S.M., Hippler, M., and Merchant, S. 2002. Reciprocal expression of two candidate di-iron enzymes affecting photosystem I and light-harvesting complex accumulation. Plant Cell 14: 673-688.

Proudfoot, N.J. 1986. Transcriptional interference and termination between duplicated $\alpha$-globin gene constructs suggests a novel mechanism for gene regulation. Nature 322: 562-565.

Rank, G., Prestel, M., and Paro, R. 2002. Transcription through intergenic chromosomal memory elements of the Drosophila bithorax complex correlates with an epigenetic switch. Mol. Cell. Biol. 22: 8026-8034.

Ren, B., Robert, F., Wyrick, J.J., Aparicio, O., Jennings, E.G., Simon, I., Zeitlinger, J., Schreiber, J., Hannett, N., Kanin, E., et al. 2000. Genome-wide location and function of DNA binding proteins. Science 290: 2306-2309.

Roberts, S.M. and Winston, F. 1996. SPT20/ADA5 encodes a novel protein functionally related to the TATA-binding protein and important for transcription in Saccharomyces cerevisiae. Mol. Cell. Biol. 16: 3206-3213.

- 1997. Essential functional interactions of SAGA, a Saccharomyces cerevisiae complex of Spt, Ada, and Gcn5 proteins, with the Snf/Swi and Srb/mediator complexes. Genetics 147: 451-465.

Sabet, N., Tong, F., Madigan, J.P., Volo, S., Smith, M.M., and Morse, R.H. 2003. Global and specific transcriptional repression by the histone $\mathrm{H} 3$ amino terminus in yeast. Proc. Natl. Acad. Sci. 100: 4084-4089.

Saha, S., Sparks, A.B., Rago, C., Akmaev, V., Wang, C.J., Vogelstein, B., Kinzler, K.W., and Velculescu, V.E. 2002. Using the transcriptome to annotate the genome. Nat. Biotechnol. 20: 508-512.

Sanchez-Herrero, E. and Akam, M. 1989. Spatially ordered transcription of regulatory DNA in the bithorax complex of Drosophila. Development 107: 321-329.

Schmitt, S., Prestel, M., and Paro, R. 2005. Intergenic transcription through a Polycomb group response element counteracts silencing. Genes \& Dev. 19: 697-708.

Sellick, C.A. and Reece, R.J. 2003. Modulation of transcription factor function by an amino acid: Activation of Put3p by proline. EMBO J. 22: 5147-5153.

- 2005. Eukaryotic transcription factors as direct nutrient sensors. Trends Biochem. Sci. 30: 405-412.

Shearwin, K.E., Callen, B.P., and Egan, J.B. 2005. Transcriptional interference-A crash course. Trends Genet. 21: 339345.

Sterner, D.E., Grant, P.A., Roberts, S.M., Duggan, L.J., Belotserkovskaya, R., Pacella, L.A., Winston, F., Workman, J.L., and Berger, S.L. 1999. Functional organization of the yeast SAGA complex: Distinct components involved in structural integrity, nucleosome acetylation, and TATA-binding protein interaction. Mol. Cell. Biol. 19: 86-98.

Storz, G., Altuvia, S., and Wassarman, K.M. 2005. An Abundance of RNA Regulators. Annu. Rev. Biochem. 74: 199217.

Sudarsanam, P., Iyer, V.R., Brown, P.O., and Winston, F. 2000. Whole-genome expression analysis of snf/swi mutants of Saccharomyces cerevisiae. Proc. Nat1. Acad. Sci. 97: 33643369.

Warfield, L., Ranish, J.A., and Hahn, S. 2004. Positive and negative functions of the SAGA complex mediated through interaction of Spt8 with TBP and the N-terminal domain of TFIIA. Genes \& Dev. 18: 1022-1034.

Winston, F. and Minehart, P.L. 1986. Analysis of the yeast SPT3 gene and identification of its product, a positive regulator of Ty transcription. Nucleic Acids Res. 14: 6885-6900.

Winston, F. Dollard, C., and Ricupero-Hovasse, S.L. 1995. Construction of a set of convenient Saccharomyces cerevisiae strains that are isogenic to S288C. Yeast 11: 53-55.

Wyers, F., Rougemaille, M., Badis, G., Rousselle, J.C., Dufour, M.E., Boulay, J., Regnault, B., Devaux, F., Namane, A., Seraphin, B., et al. 2005. Cryptic pol II transcripts are degraded by a nuclear quality control pathway involving a new poly(A) polymerase. Cell 121: 725-737. 


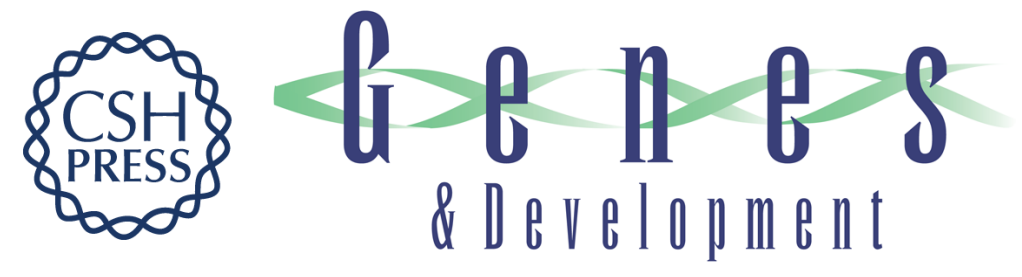

\section{Regulation of an intergenic transcript controls adjacent gene transcription in Saccharomyces cerevisiae}

Joseph A. Martens, Pei-Yun Jenny Wu and Fred Winston

Genes Dev. 2005, 19:

Access the most recent version at doi:10.1101/gad.1367605

Supplemental http://genesdev.cshlp.org/content/suppl/2005/10/27/19.22.2695.DC1
Material

References This article cites 67 articles, 39 of which can be accessed free at:

http://genesdev.cshlp.org/content/19/22/2695.full.html\#ref-list-1

License

Email Alerting Receive free email alerts when new articles cite this article - sign up in the box at the top

Service

right corner of the article or click here.

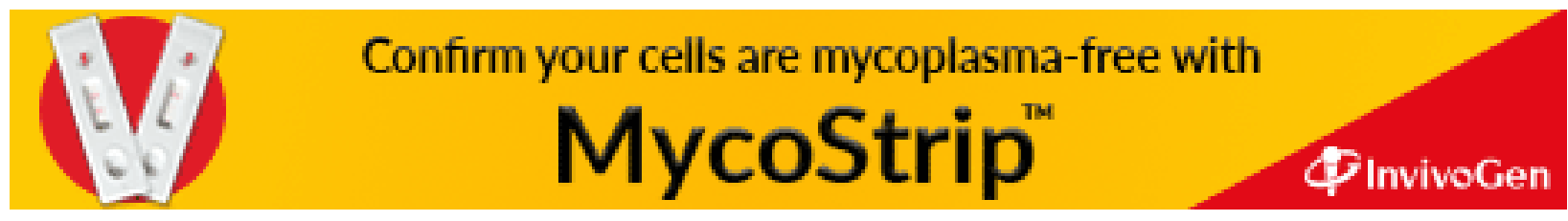

